\title{
Magnetization measurements of high-mobility two-dimensional electron gases
}

\author{
M. Zhu, ${ }^{1}$ A. Usher, ${ }^{1}$ A. J. Matthews, ${ }^{1}$ A. Potts, ${ }^{2, *}$ M. Elliott, ${ }^{2}$ W. G. Herrenden-Harker, ${ }^{2}$ D. A. Ritchie, ${ }^{3}$ \\ and M. Y. Simmons ${ }^{3, \dagger}$ \\ ${ }^{1}$ School of Physics, University of Exeter, Stocker Road, Exeter EX4 4QL, United Kingdom \\ ${ }^{2}$ Department of Physics and Astronomy, Cardiff University, 5 The Parade, Cardiff CF24 3YB, United Kingdom \\ ${ }^{3}$ Cavendish Laboratory, Madingley Road, Cambridge CB3 OHE, United Kingdom
}

(Received 22 April 2002; published 30 April 2003)

\begin{abstract}
De Haas-van Alphen (dHvA) oscillations are observed for Landau levels (LLs) with filling factors between 4 and 52 , at temperatures in the range $50 \mathrm{mK}$ to $1 \mathrm{~K}$, in experiments on high-mobility $\mathrm{GaAs} /(\mathrm{Al}, \mathrm{Ga}) \mathrm{As}$ heterojunctions. The oscillations become sawtooth-shaped at low filling factors, and theoretical fits to the data, assuming the two-dimensional electron gas to be a non-interacting Fermi system, show the shape of LLs to be close to a $\delta$ function. The small residual width $(\sim 0.4 \mathrm{meV}$ or less $)$ fits equally well to either a Gaussian or a Lorentzian density-of-states model. In almost all cases, a constant background density of states has to be included to obtain a satisfactory fit. Weak odd-filling-factor dHvA peaks are detected at high fields, from which a $g$-factor enhancement of 15 can be inferred. Comparison of the scattering time derived from the fits before and after illumination, with the momentum relaxation time derived from transport, reveals a counterintuitive behavior in the bulk-modulation-doped sample.
\end{abstract}

DOI: 10.1103/PhysRevB.67.155329

PACS number(s): 73.43.Fj, 73.40.Kp, 73.20.At, 75.20.-g

\section{INTRODUCTION}

A knowledge of the density of states (DOS) of twodimensional electron systems (2DES) subjected to high magnetic fields is central to our understanding of such systems. ${ }^{1-5}$ Techniques used to measure the DOS have included photoluminescence, ${ }^{6}$ magnetocapacitance,${ }^{7-9}$ specific heat,${ }^{10-12}$ and magnetization. ${ }^{13-17}$ Perhaps the most promising of these is magnetization, because it represents a minimal perturbation to the system and is relatively easy to analyze.

In a perpendicular magnetic field $B$, the DOS of an ideal two-dimensional electron gas will split into a series of Landau levels (LLs) which are of the form of $\delta$ functions, separated by $\hbar \omega_{c}$. Here $\omega_{c}=e B / m^{*}$ is the cyclotron frequency of an electron in the magnetic field. The magnetization is predicted to oscillate periodically in a sawtooth manner as a function of filling factor $\nu$, the de Haas-van Alphen (dHvA) effect. For an isolated 2DES the sharp jump occurs on the high field side of each sawtooth. In reality the degeneracy of each LL will be lifted because of impurity scattering and the electron-electron interaction, and information about these effects can be obtained by measuring the shape of the DOS, from the deviation of the $\mathrm{dHvA}$ oscillations from their ideal sawtooth form.

Theories have suggested Gaussian ${ }^{18}$ or elliptical ${ }^{19}$ shapes for the LL DOS, and the short-range scattering theory of Aoki and Ando ${ }^{1}$ predicted that the width $\Gamma$ is proportional to $B^{1 / 2}$. However, early experiments found the LLs to be Gaussian, but with widths larger than theoretical predictions. ${ }^{8,10}$ Some of these experiments suggested that $\Gamma$ is $B$ independent, ${ }^{10,13,16}$ while others argued that $\Gamma \propto B^{1 / 2}, 8,9,14$ as the theory of Aoki and Ando predicted, or that $\Gamma$ is related to $B$ in a more complicated way. ${ }^{6,11,12}$ Lorentzian broadened LLs were also proposed. ${ }^{17}$ Das Sarma and $\mathrm{Xie}^{4}$ introduced a model, based on the theory of Ando and Murayama, ${ }^{2}$ which takes account of long-range scattering when the Fermi energy lies in the tail of a LL by including self-consistent screening and LL coupling. A wider and $B$-dependent LL width was predicted, which was consistent with some of the earlier experimental studies. $8,9,14$

Apart from the shape of the LLs, the existence of an absolute gap between two adjacent LLs was also questioned. It was found that a constant DOS background had to be introduced to resolve the discrepancy between the experimental results and theory (e.g., Refs. 8, 9, 13, and 14). The background was argued to be the result of the long range of scattering $^{6}$ and/or the presence of inhomogeneities. ${ }^{20}$

Extensive studies of dHvA oscillations in the magnetization of 2DESs have been carried out theoretically ${ }^{21-24}$ and experimentally ${ }^{13-17,21,25,26}$ since the discovery of the integer quantum Hall effect. Störmer et $_{\text {al. }}{ }^{2}$ made the first convincing measurement of dHvA oscillations in a 2DES by using a dc superconducting quantum interference device (SQUID) magnetometer. The shape of the LLs has since been examined quantitatively in the detailed torque-magnetometry studies of Potts et al. ${ }^{17}$ which suggested that the DOS consists of a series of Lorentzian-broadened LLs with $\Gamma$ independent of $B$. At lower fields they found that Gaussian-shaped LLs with a width of $\Gamma \propto B^{1 / 2}$ gave indistinguishable numerical fitting results.

Most of these early dHvA measurements were performed on multi-layer samples in order to enhance the extremely weak dHvA signal. As a result, the line shapes and widths obtained may be influenced by interlayer inhomogeneities. A dramatic improvement in the sensitivity of the torque magnetometry technique in the last few years has enabled measurements to be performed on single-layer samples. Wiegers et al. ${ }^{27}$ reported sawtooth $\mathrm{dHvA}$ oscillations detected in higher mobility single-layer samples. Using a modulated SQUID technique, Meinel et al. ${ }^{28}$ observed similar sharp dHvA oscillations. However, no quantitative analysis of the LL shape of single-layer 2DESs has yet been reported.

In this paper we report the results of magnetization measurements performed on $\delta$-modulation-doped and bulk- 
TABLE I. Summary of results.

\begin{tabular}{|c|c|c|c|c|c|c|c|c|c|}
\hline \multirow[b]{2}{*}{ Sample } & \multirow[b]{2}{*}{$\begin{array}{l}\text { Illumination } \\
\text { state }\end{array}$} & \multicolumn{2}{|c|}{ Transport, $4.2 \mathrm{~K}$} & \multirow[b]{2}{*}{$T(\mathrm{mK})$} & \multicolumn{5}{|c|}{ Fitting parameters } \\
\hline & & $\begin{array}{c}n_{e} \\
\left(\times 10^{15} \mathrm{~m}^{-2}\right)\end{array}$ & $\begin{array}{c}\mu \\
\left(\mathrm{m}^{2} / \mathrm{Vs}\right)\end{array}$ & & Model & $\begin{array}{c}n_{e} \\
\left(\times 10^{15} \mathrm{~m}^{-2}\right)\end{array}$ & $\begin{array}{c}\Gamma \\
(\mathrm{meV}) \\
\pm 0.05\end{array}$ & $\begin{array}{c}\xi \\
\pm 0.15\end{array}$ & $\begin{array}{c}S \\
\pm 0.05\end{array}$ \\
\hline \multirow[t]{4}{*}{$\mathrm{T} 73$} & Before & 4.4 & 50 & 50 & Gauss & 4.48 & 0.28 & 0.31 & 1.10 \\
\hline & & & & & Lorentz & 4.48 & 0.25 & 0.04 & 1.10 \\
\hline & After & 8.6 & 69 & 50 & Gauss & 8.15 & 0.27 & 0.14 & 1.10 \\
\hline & & & & & Lorentz & 8.15 & 0.10 & 0.11 & 1.10 \\
\hline \multirow[t]{6}{*}{$\mathrm{T} 412$} & Before & 3.1 & 78 & 190 & Gauss & 3.05 & 0.23 & 0.49 & 1.05 \\
\hline & & & & & Lorentz & 3.05 & 0.22 & 0.28 & 1.10 \\
\hline & After & 4.9 & 130 & 540 & Gauss & 4.98 & 0.39 & 0.23 & 1.05 \\
\hline & & & & & Lorentz & 4.98 & 0.34 & 0.00 & 1.10 \\
\hline & & & & 1000 & Gauss & 4.98 & 0.37 & 0.22 & 1.05 \\
\hline & & & & & Lorentz & 4.98 & 0.33 & 0.00 & 1.10 \\
\hline
\end{tabular}

modulation-doped GaAs/(Al, Ga)As heterojunctions at temperatures between $50 \mathrm{mK}$ and $1 \mathrm{~K}$. Clear sawtooth dHvA oscillations in the magnetization are observed for LLs of filling factors between 4 and 52. The large number of wellresolved oscillations enables us to accurately estimate the LL linewidth, where a Gaussian or a Lorentzian DOS model is assumed.

It is well known that illuminating a sample changes the transport properties of a $2 \mathrm{DES}$, usually increasing both the number of electrons in the 2DES and their mobility. In this paper we report the effects of illumination on the 2DES's thermodynamic properties and show that they are strongly dependent on the profile of the modulation doping: whether the sample is bulk- or $\delta$-modulation doped.

\section{EXPERIMENTAL DETAILS}

The experiments were performed on a high-mobility $\delta$-modulation-doped $\mathrm{GaAs} /(\mathrm{Al}, \mathrm{Ga}) \mathrm{As}$ sample designated T73 and a bulk-modulation-doped sample T412. Both samples were uncontacted squares of side $10 \mathrm{~mm}$. The transport parameters electron density $n_{e}$ and mobility $\mu$ for the two samples, measured at $4.2 \mathrm{~K}$ before and after illumination, are summarized in Table I.

The sample was mounted on a torque magnetometer. Details of the technique have been described elsewhere. ${ }^{29}$ The experiment was carried out in the mixing chamber of a dilution refrigerator with a base temperature of less than $50 \mathrm{mK}$. Magnetic fields of up to $15.5 \mathrm{~T}$ were applied, swept at rates from $1.87 \times 10^{-4}$ to $2.4 \times 10^{-4} \mathrm{~T} / \mathrm{s}$. The normal to the $2 \mathrm{DES}$ plane was tilted at $20^{\circ}$ with respect to the magnet field direction. The temperatures were controlled within the range $50 \mathrm{mK}$ to $1 \mathrm{~K}$, and measured using a calibrated Ruthenium Oxide chip resistor mounted on the magnetometer. The illumination was carried out by exposure to red and/or infrared radiation from light-emitting diodes, until the change in $n_{e}$ saturated. A coil on the magnetometer rotor provided an approximate calibration of the instrument's sensitivity $( \pm 10 \%)$, which was used as a starting point for the fitting described below.

\section{RESULTS AND DISCUSSION}

\section{A. Extraction of dHvA oscillations from the raw data}

Magnetization data were taken while the magnetic field was swept in both directions and then added together and divided by two, to minimize systematic errors and to cancel eddy currents. The latter are induced by sweeping the magnetic field and reverse when the magnetic-field sweep direction is reversed. The non-equilibrium magnetization caused by eddy currents is up to 50 times larger than the dHvA signal for low filling factors at $50 \mathrm{mK}$ but is much less robust to elevated temperature, disappearing completely by about 1 $\mathrm{K}$. The raw magnetization signal also includes a large slowly varying background, caused by magnetic impurities in the magnetometer rotor. ${ }^{17,27}$ This background was removed using the following procedure: A polynomial of up to sixth order was subtracted from the raw data to remove most of the background; then the remainder was removed by assuming that the mean position of the dHvA oscillations is independent of field. In the few cases when the cancellation of the eddy currents was incomplete, the residual eddy current was ignored in the estimation of this mean position.

The dHvA oscillations are sharp and sawtooth-shaped at high fields and low temperatures (Fig. 1), which indicates that the 2DES is close to an ideal system and that the LLs are very narrow. However the sawtooth observed is not infinitely steep on the high-magnetic-field side as expected for a 2DES with fixed $n_{e}$, having $\delta$-function Landau levels separated by energy gaps. The possible causes of this are discussed below.

\section{B. The model}

A numerical fitting was carried out based on the method reported by Potts et al., ${ }^{17}$ in which the DOS is assumed to have the form

$$
D(B, E)=\xi \frac{m^{*}}{\pi \hbar^{2}}+(1-\xi) \frac{2 e B}{\pi h} g(E) .
$$

The first term represents a constant background DOS, and the second term is the DOS of the LLs. $\xi$ is the proportion of 


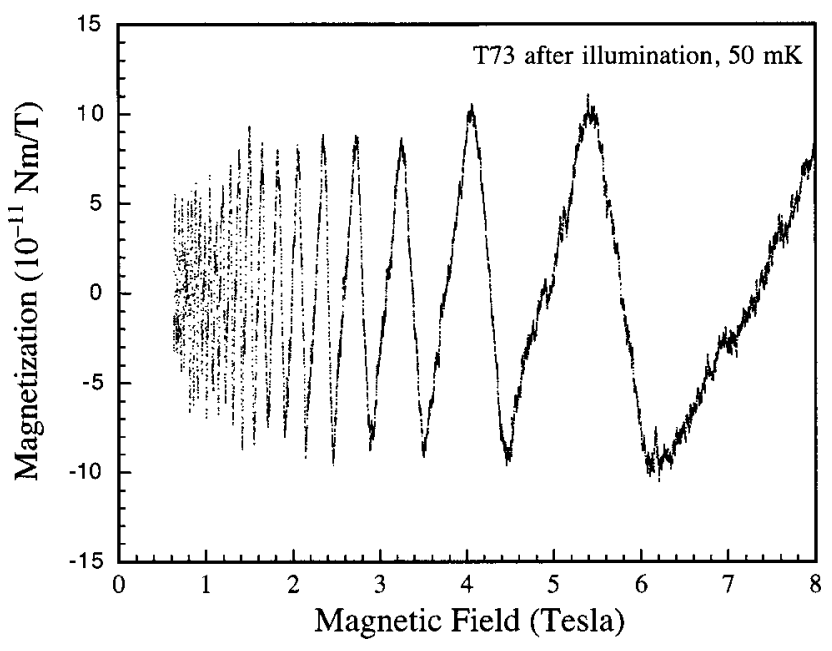

FIG. 1. The magnetization of the $\delta$-modulation-doped sample T73 recorded at $50 \mathrm{mK}$ after illumination, as a function of the perpendicular magnetic field. The oscillations become sawtooth in shape at high field, but neither side of the sawtooth is abrupt, for reasons discussed in the text.

states making up the background, and $g(E)$ describes the shape of the LLs. We model the LLs as being either Gaussian,

$$
g(E)=\frac{1}{\pi \ell^{2}} \frac{1}{\sqrt{2 \pi} \Gamma} \sum_{n=0}^{\infty} \exp \left(-\frac{\left(E-E_{n}\right)^{2}}{2 \Gamma^{2}}\right),
$$

or Lorentzian,

$$
g(E)=\frac{1}{(\pi \ell)^{2}} \sum_{n=0}^{\infty} \frac{\Gamma}{\left(E-E_{n}\right)^{2}+\Gamma^{2}},
$$

in shape, where $\Gamma$ is a width parameter. $\ell=(\hbar / e B)^{1 / 2}$ is the Landau radius, $E_{n}=(n+1 / 2) \hbar \omega_{c}$ is the energy of the $n$th $\mathrm{LL}$, and $\omega_{c}=e B / m^{*}$ is the cyclotron frequency. $\Gamma$ was allowed to take the form $\Gamma(B)=\Gamma_{0} B^{p}$ with $p=0$, or $p=\frac{1}{2}$, but the field-independent form was found always to produce the best fits for both Lorentzian and Gaussian models, so the $p$ $=\frac{1}{2}$ case will not be discussed any further. This observation is consistent with that of Potts et al. ${ }^{17}$ that a fieldindependent Lorentzian gave the best overall fit, but not with their finding that a field-dependent Gaussian gave an equally good fit at low fields.

Assuming that the 2DES is an ideal non-interacting Fermi system, in which spin splitting is ignored, the thermodynamic potential per unit area $\Omega$ at the temperature $T$ is given by

$$
\Omega(B, T)=k T \int_{0}^{\infty} d E D(B, E) \ln \left(1-\frac{1}{1+\exp \left(\frac{E-E_{F}}{k T}\right)}\right),
$$

where $E_{F}$ is the Fermi energy. Under the assumption that the electron density $n_{e}$ is fixed, the energy $\Omega$ is determined by the position of the Fermi energy, which oscillates with the magnetic field applied. The magnetization of the 2DES is given by

$$
M(B, T)=-\left.\frac{\partial \Omega(B, T)}{\partial B}\right|_{E_{F}, T} .
$$

The calculated magnetization using the two model DOSs is fitted to the experimental data, treating $n_{e}, \Gamma$ and $\xi$ as fitting parameters, and assuming an electron effective mass of $0.0667 m_{e}$. A scaling factor $S$ is applied to $M$ and allowed to vary within $\pm 10 \%$ of the calibration obtained from the calibration coil. The effects of these parameters on the shape of the fitted curve are as follows: $n_{e}$ controls the frequency of the $1 / B$ oscillations; $S$ controls their amplitude; $\xi$ controls the relative slopes of the two sides of the sawtooth, particularly noticeable at high magnetic fields (when $\xi=0$ the high-field side has infinite slope), and also the amplitude (consequently $S$ and $\xi$ cannot both be treated as free fitting parameters in the same fit); and $\Gamma$ controls the rate of damping of the oscillations as $B \rightarrow 0$, and has a slight smoothing effect on the sawtooth towards a sinusoidal form, an effect most visible at low fields. Three parameter fits of $n_{e}, \Gamma$ and $\xi$ or $n_{e}, \Gamma$ and $S$ produce reliable and repeatable results thanks largely to the large number of oscillations present in the data, from these millikelvin temperature experiments.

\section{Numerical fitting results}

Figures 2 and 3 show typical dHvA data for the two samples and the results of fits using Gaussian and Lorentzian broadening. For clarity, only one fit is shown per plot but the results of all the fits are summarized in Table I. The fitted carrier densities are in close agreement with those obtained from transport. No significant improvement in the fit was obtained by allowing population of the second twodimensional subband, for any of the data presented. To confirm this, the data (interpolated to be evenly spaced in $1 / B$ ) were Fourier transformed. In all cases the Fourier transforms [an example is shown in the inset to Fig. 2(b)] consisted of a single fundamental field plus harmonics; the carrier densities obtained from these were in close agreement with the fitted densities in Table I.

\section{Discussion}

\section{Density of states}

The LL widths summarized in Table I $(0.1-0.4 \mathrm{meV})$ are much smaller than in the study of Potts et al.,${ }^{17}$ demonstrating the improved quality of these heterojunctions. The LL shape is described equally well by a Gaussian or a Lorentzian function, with a width independent of magnetic field, the quality of the different fits to each data set never differing by more than $6 \%$. This is consistent with Potts et al., who found the best fit to be Lorentzian, $B$ independent. The independence of the width on $B$ also supports the observations of Refs. 7, 10, 13, and 16. In almost all cases in Table I we have to invoke a significant background density of states between LLs which is larger when the Gaussian shape is assumed. 


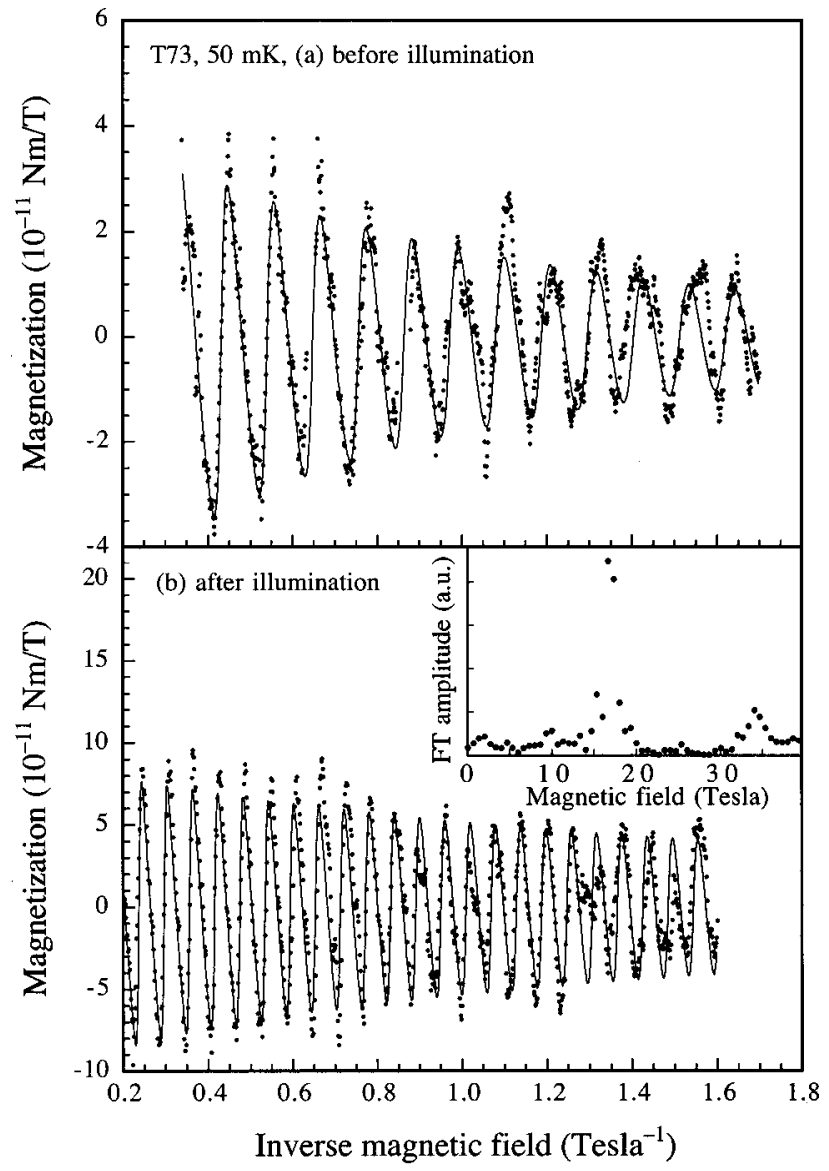

FIG. 2. The magnetization of the $\delta$-modulation-doped sample $\mathrm{T} 73$, as a function of inverse magnetic field recorded at $50 \mathrm{mK}$, (a) before illumination, and (b) after illumination. The lines are theoretical fits to the data using the Lorentzian model density of states. Values of the fitting parameters are given in Table I. The inset shows the result of a discrete Fourier transform of the data in (b). Peaks at the fundamental field $16.7( \pm 0.3) \mathrm{T}$, and its first harmonic, are consistent with the fitted value of $n_{e}$.

The proportion of states in this background, up to $49 \%$, is consistent with some of the earlier experiments in which the background was attributed to the impurities and sample inhomogeneity. ${ }^{6,8-10,16}$ However, it contrasts with the findings of Potts et al., suggesting either that the constant background is associated with the higher sample quality, or more likely that the relatively broad Lorentzians used to fit the data of Potts et al. include a large number of states between LLs without the need to introduce a background. The need for less background in the Lorentzian case might also incline us tentatively to favor this model. It should be noted that no amount of adjustment of the other parameters can yield a satisfactory fit to the data-only the inclusion of a non-zero $\xi$ achieves this.

As mentioned earlier, the dHvA oscillations are sawtooth in shape at high $B$ in these high-quality structures. The background density of states is introduced into the model in order to fit the non-abrupt rise on the high- $B$ side of the sawtooth seen in the data. However there is another possible cause of this feature of the data: an electron density $n_{e}$, which oscillates with $B$. This could occur if the 2DES were not isolated,

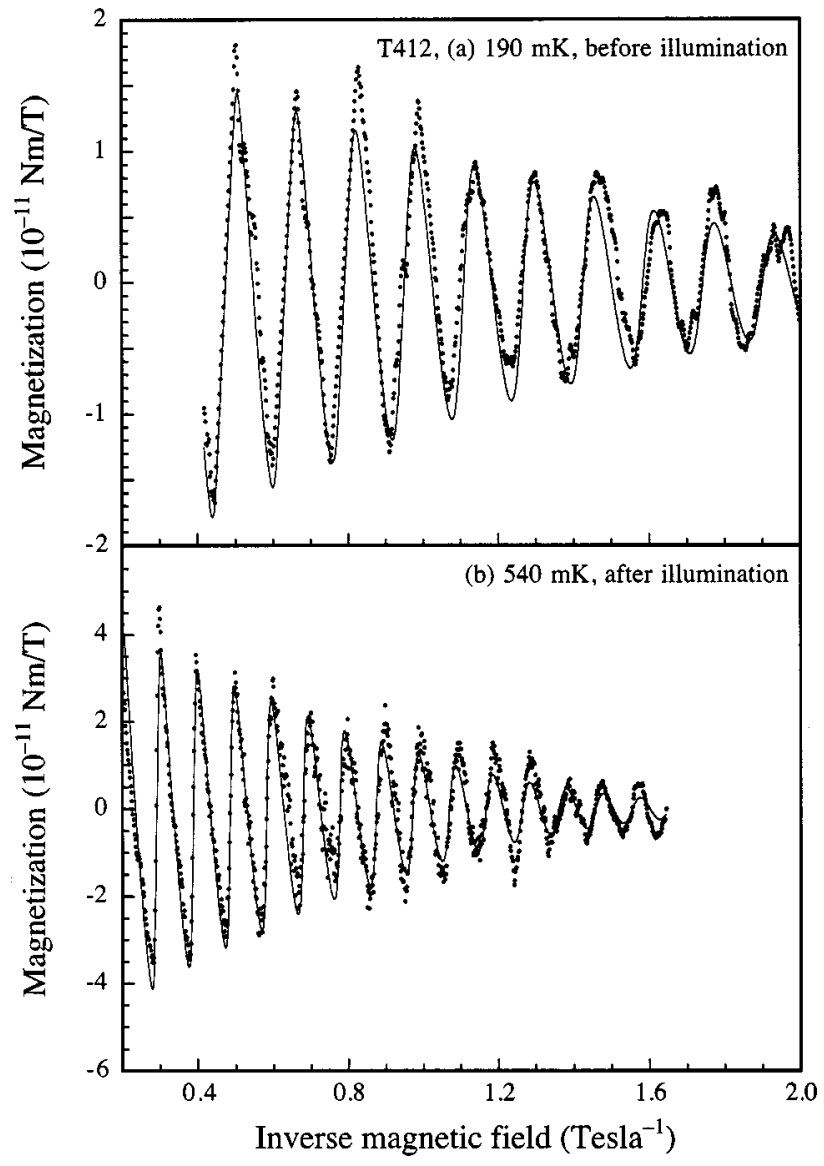

FIG. 3. The magnetization of the bulk-modulation-doped sample T412, as a function of inverse magnetic field (a) before illumination at $190 \mathrm{mK}$, and (b) after illumination at $540 \mathrm{mK}$. The lines are theoretical fits to the data using the Gaussian model density of states. Values of the fitting parameters are given in Table I.

but instead were connected to a large reservoir of electrons (in other words if the 2DES formed a grand canonical rather than a canonical ensemble). This possibility was proposed by Shoenberg. ${ }^{22}$ In this case, the system is constrained to have constant $E_{F}$, rather than constant $n_{e}$, and the result is that the abrupt jump in the dHvA oscillation moves to the low magnetic field side of the sawtooth. This has been confirmed by Meinel $e t a .^{28}$ in an experiment in which $n_{e}$ is modulated by applying a gate voltage to the sample. We do not observe exactly this behavior in our experiments: both sides of the sawtooth are nonabrupt, implying some variation of both $n_{e}$ and $E_{F}$. The mechanism by which electrons could move into and out of the 2DES in our noncontacted samples is not known, although we note that the in-plane component of $B$ might assist a tunnelling process between the 2DES and the doped (Al, Ga)As, an effect invoked by Boebinger et al. ${ }^{30}$ to explain the enhanced coupling between parallel 2DESs in a double-quantum-well structure, caused by an in-plane magnetic field. Recent observations of oscillations in the recombination energy of electrons with photoexcited holes in a $\delta$-modulation-doped heterojunction ${ }^{31}$ and analysis of cyclotron resonance data ${ }^{32}$ have also been interpreted in terms of such a transfer of charge. 


\section{Effects of illumination}

It is well established from transport measurements that illumination increases both $n_{e}$ and $\mu$, through the phenomenon of persistent photoconductivity. In this section we examine for the first time the effects of illumination on the thermodynamic DOS of the LLs, and compare these with transport measurements on the same samples. The fundamental difference between thermodynamic and transport studies is that the former measure the properties of all the states in the system, while the latter are sensitive only to extended states.

From Table I, and from a visual inspection of the damping of the data in $1 / B$, we find that the LL width of $\mathrm{T} 73$ is reduced, while the mobility has increased, suggesting that illumination has affected the extended states and localized states in a broadly similar way. However, in T412 no such similarity is found: the mobility increases by $67 \%$ on illumination, but the fitted LL width actually increases, by about $62 \%$, an effect that once again is evident from a visual inspection of the raw data. This surprising result underlines the fact that extended and localized states in these systems do in general sample the disorder in very different ways-in this case the illumination has reduced the disorder experienced by the extended states (the only type of state influencing the transport mobility), while increasing that experienced by the localized states (to which dHvA measurements are sensitive).

We also observe that the induced eddy currents in both samples virtually disappear after illumination. We suggest that this is because the increased mobility increases the proportion of the states in the LL that are extended, and hence narrows the Hall plateaus and magnetoresistance minima, weakening the eddy currents.

\section{Odd filling factors}

We expect to see dHvA oscillations due to the spin splitting of the Landau levels at high magnetic fields. However, we find that these weak oscillations are masked by the induced eddy currents in both samples before illumination, but are visible after illumination. Figure 4 shows the odd-filling factor oscillations at $\nu=3$ and 5 in T412 after illumination. An estimate of the exchange-enhanced $g$-factor can be made by comparing the magnetization jump at $\nu=3$ with that at $\nu=4$ (for which the energy gap is assumed to be $\hbar \omega_{c}$ ). This yields $g * \sim 4.8$, enhanced by some 11 times compared with its bare value, but still somewhat lower than the value of 7.3 reported in activated transport studies of exchange enhancement. ${ }^{33}$ However if we follow the suggestion of that study that the energy gap at even filling factors is also enhanced, by about $33 \%$ in samples of similar mobilities to ours, then we estimate $g *$ to be 6.4 .

The odd-filling factor dHvA features in T73 are significantly weaker, giving $g^{*} \sim 2$. This is probably a reflection of

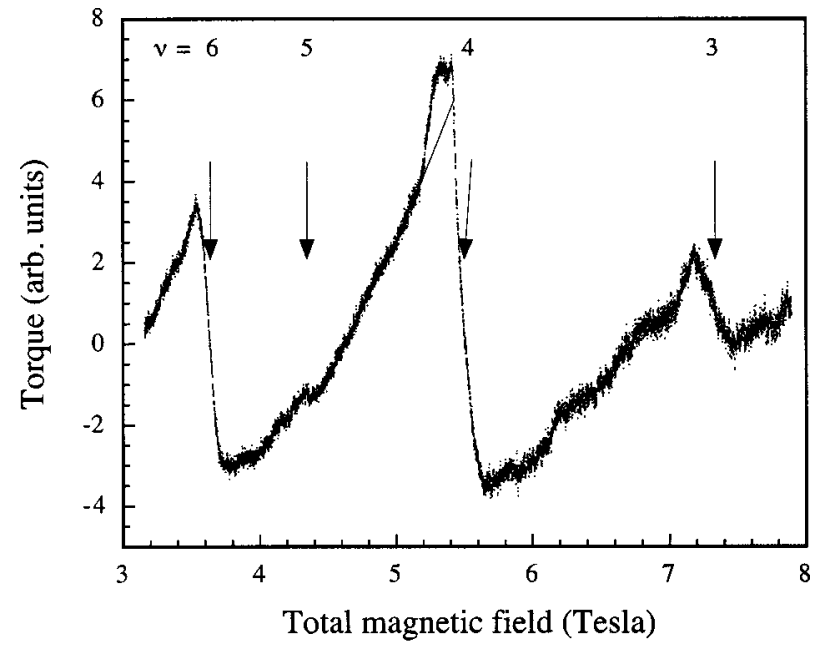

FIG. 4. Raw data for T412, showing dHvA features at odd filling factors 3 and 5, from which the exchange-enhanced $g$ factor can be calculated. The data were recorded at $540 \mathrm{mK}$ after illumination. Under these conditions induced eddy currents are suppressed, although the distortion of the sawtooth shape around $\nu=4$ is the remnant of an eddy current.

the lower mobility of this sample, but contrasts strongly with the observation of Wiegers et al. ${ }^{27}$ that the odd dHvA peaks were as strong as the even ones in a $\delta$-modulation-doped heterojunction similar to T73.

\section{CONCLUSIONS}

We have observed de Haas-van Alphen oscillations in two high-mobility heterojunctions, at filling factors up to 52, which tend towards the sawtooth shape of ideal $\delta$-function Landau levels at high fields. The data fit equally well to Gaussian or Lorentzian broadened density-of-states models with widths independent of magnetic field and temperature, and these widths are in the range $0.1-0.4 \mathrm{meV}$. In most cases a constant background density of states between the levels (of up to $49 \%$ ) has to be introduced to produce a satisfactory fit, the Lorentzian model requiring less background than the Gaussian. After illumination, both samples show the expected increase in mobility and number density due to persistent photoconductivity, and, in the $\delta$-modulation-doped sample this is accompanied by a reduction in the fitted width. Unexpectedly, though, the bulk modulation doped sample shows an increase in width after illumination. Finally, we observe de Haas-van Alphen oscillations at small odd filling factors in both samples after illumination has removed the induced eddy currents. Comparing the size of these with those at neighboring even filling factors, we deduce that the electron $g$ factor is exchange-enhanced from its bare value by up to 15 times.
*Present address: Department of Electronics and Computer Science, University of Southampton, Southampton SO17 1BJ, United Kingdom.

†Present address: School of Physics, The University of New South
Wales, Sydney 2052, Australia.

${ }^{1}$ H. Aoki and T. Ando, Solid State Commun. 38, 1079 (1981).

${ }^{2}$ T. Ando and Y. Murayama, J. Phys. Soc. Jpn. 54, 1519 (1985).

${ }^{3}$ K. von Klitzing, Rev. Mod. Phys. 58, 519 (1986). 
${ }^{4}$ S. Das Sarma and X. C. Xie, J. Appl. Phys. 64, 5465 (1988).

${ }^{5}$ Q. Li, X. C. Xie, and S. Das Sarma, Phys. Rev. B 40, 1381 (1989).

${ }^{6}$ T. T. J. M. Berendschot, H. A. J. M. Reinmen, and H. J. A. Bluyssen, Solid State Commun. 63, 873 (1987).

${ }^{7}$ T. P. Smith, B. B. Goldberg, P. J. Stiles, and M. Heiblum, Phys. Rev. B 32, 2696 (1985).

${ }^{8}$ V. Mosser, D. Weiss, K. von Klitzing, K. Ploog, and G. Weimann, Solid State Commun. 58, 5 (1986).

${ }^{9}$ H. Zheng, A. Song, F. Yang, and Y. Li, Phys. Rev. B 49, 1802 (1994).

${ }^{10}$ E. Gornik, R. Lassnig, and G. Strasser, Phys. Rev. Lett. 54, 1820 (1985).

${ }^{11}$ J. K. Wang, J. H. Campbell, D. C. Tsui, and A. Y. Cho, Phys. Rev. B 38, 6174 (1988).

${ }^{12}$ J. K. Wang, D. C. Tsui, M. Santos, and M. Shayegan, Phys. Rev. B 45, 4384 (1992).

${ }^{13}$ T. Haavasoja, H. L. Störmer, D. J. Bishop, V. Narayanamurti, A. C. Gossard, and W. Wiemann, Surf. Sci. 142, 294 (1984).

${ }^{14}$ J. P. Eisenstein, H. L. Störmer, V. Narayanamurti, A. Y. Cho, A. C. Gossard, and C. W. Tu, Phys. Rev. Lett. 55, 875 (1985).

${ }^{15}$ J. P. Eisenstein, Appl. Phys. Lett. 46, 695 (1985).

${ }^{16}$ I. M. Templeton, J. Appl. Phys. 64, 3570 (1988).

${ }^{17}$ A. Potts, R. Shepherd, W. G. Herrenden-Harker, M. Elliott, C. L. Jones, A. Usher, G. A. Jones, D. A. Ritchie, E. H. Linfield, and M. Grimshaw, J. Phys. C 8, 5189 (1996).

${ }^{18}$ F. Wegner, Z. Phys. B 51, 279 (1983).

${ }^{19}$ T. Ando and Y. Uemura, J. Phys. Soc. Jpn. 36, 959 (1974).
${ }^{20}$ R. R. Gerhardts and V. Gudmundsson, Phys. Rev. B 34, 2999 (1986).

${ }^{21}$ H. L. Störmer, T. Haavasoja, V. Narayanamurti, A. C. Gossard, and W. Wiemann, J. Vac. Sci. Technol., B 1, 423 (1983).

${ }^{22}$ D. Shoenberg, J. Low Temp. Phys. 56, 417 (1984).

${ }^{23}$ R. Haussman, Phys. Rev. B 56, 9684 (1997).

${ }^{24}$ L. Bremme, T. Ihn, and K. Ensslin, Phys. Rev. B 59, 7305 (1999).

${ }^{25}$ F. F. Fang, Surf. Sci. 142, 290 (1984).

${ }^{26}$ I. M. Templeton, J. Appl. Phys. 62, 4005 (1987).

${ }^{27}$ S. A. J. Wiegers, M. Specht, L. P. Lévy, M. Y. Simmons, D. A. Ritchie, A. Cavanna, B. Etienne, G. Martinez, and P. Wyder, Phys. Rev. Lett. 79, 3238 (1997).

${ }^{28}$ I. Meinel, T. Hengstmann, D. Grundler, H. Heitmann, W. Wegscheider, and M. Bichler, Phys. Rev. Lett. 82, 819 (1999).

${ }^{29}$ C. L. Jones, A. Usher, M. Elliott, W. G. Herrenden-Harker, A. Potts, R. Shepherd, T. S. Cheng, and C. T. Foxon, Solid State Commun. 95, 409 (1995).

${ }^{30}$ G. S. Boebinger, A. Passner, L. N. Pfeiffer, and K. W. West, Phys. Rev. B 43, 12673 (1991).

${ }^{31}$ G. C. Kerridge, M. G. Greally, M. Hayne, A. Usher, A. S. Plaut, J. A. Brum, M. C. Holland, and C. R. Stanley, Solid State Commun. 109, 267 (1999).

${ }^{32}$ A. Raymond, S. Juillaguet, I. Elmezouar, W. Zawadzki, M. L. Sadowski, M. Kamal-Saadi, and B. Etienne, Semicond. Sci. Technol. 14, 915 (1999).

${ }^{33}$ A. Usher, R. J. Nicholas, J. J. Harris, and C. T. Foxon, Phys. Rev. B 41, 1129 (1990). 